\title{
A Learners Experience with the Games Education in Software Engineering
}

DOI

\section{https://doi.org/10.1007/978-3-030-52190-5 27}

\section{Authors}

Muhammad Imran Tariq, Jorge Diaz-Martinez, Shariq Aziz Butt, Muhammad Adeel, Emiro De-la-Hoz-Franco, Anca Mihaela Dicu

\begin{abstract}
The utilization of games and its components in software building training isn't new. In reality, their usage in Software development training is found to look into papers for numerous decades. Be that as it may, there is little data about the real reception of these methodologies in games designing and development training in terms of software building. Consequently, the objective of this paper is to research the utilization of game's components in programming designing instruction, in the viewpoint of teachers. To accomplish this objective, this examination proposes and breaks down the aftereffects of a study replied by numerous product designing teachers. We test the members by welcoming multiple instructors from settled colleges and instructive organizations of various districts. The first objective of the review is to gather data relates to the utilization of important games and rich gamification in the student classrooms, and the second objective is to comprehend information territories and the pre-owned game-related techniques. The outcomes show that the more significant part of the educators knows about these instructive methodologies, the games were embraced by various members. Games that are generally played to cover "Programming Process" \& "Task Management." The utmost utilized game apparatuses are Points scoring, Quizzes solving, and meet challenges. The outcomes additionally reveal that the fundamental explanations behind not embracing the assets are the absence of information, lack of data about essential games for encouraging programming designing, and the absence of time to design and remember these methodologies for the classes At long last, results show a definite propensity towards the future appropriation of these game-related methodologies by the product developing teachers.
\end{abstract}

\section{Keywords}

Games design, Game development, Software engineering, Games-based learning 